\title{
Measurements of neutron-induced light-charged particle emission reactions
}

Guohui Zhang ${ }^{1, *}$, Huaiyong Bai ${ }^{1}$, Haoyu Jiang ${ }^{1}$, Zengqi Cui ${ }^{1}$, Yi $\mathrm{Lu}^{1}$, Yiwei $\mathrm{Hu}^{1}$, Jinxiang $\mathrm{Chen}^{1}$, Yu. M. Gledenov ${ }^{2}$, M. V. Sedysheva ${ }^{2}$, G. Khuukhenkhuu ${ }^{3}$, Ruirui Fan ${ }^{4,5,6}$, Wei Jiang ${ }^{5,6}$, Han Yi ${ }^{5,6}$, Jingyu Tang ${ }^{5,6}$, LiangZhou ${ }^{5,6}$, Changjun $\mathrm{Ning}^{5,6}$, Qi An ${ }^{4,7}$, Jie $\mathrm{Bao}^{8}$, Yu Bao ${ }^{5,6}$, Ping $\mathrm{Cao}^{4,7}$, Haolei Chen ${ }^{4,7}$, Qiping Chen ${ }^{9}$, Yonghao Chen ${ }^{5,6}$, Yukai Chen ${ }^{5,6}$, Zhen Chen ${ }^{4,7}$, Changqing Feng ${ }^{4,7}$, Keqing Gao ${ }^{5,6}$, Minhao Gu ${ }^{4,5}$, Changcai $\mathrm{Han}^{10}$, Zijie $\mathrm{Han}^{9}$, Guozhu $\mathrm{He}^{8}$, Yongcheng $\mathrm{He}^{5,6}$, Yang Hong ${ }^{5,6,11}$, Hanxiong Huang ${ }^{8}$, Weiling Huang ${ }^{5,6}$, Xiru Huang ${ }^{4,7}$, Xiaolu $\mathrm{Ji}^{4,5}$, Xuyang $\mathrm{Ji}^{4,12}$, Zhijie Jiang ${ }^{4,7}$, Hantao Jing ${ }^{5,6}$, Ling Kang ${ }^{5,6}$, Mingtao Kang ${ }^{5,6}$, Bo $\mathrm{Li}^{5,6}$, Chao $\mathrm{Li}^{4,7}$, Jiawen $\mathrm{Li}^{4,12}$, Lun $\mathrm{Li}^{5,6}$, Qiang Li ${ }^{5,6}$, Xiao Li ${ }^{5,6}$, Yang Li ${ }^{5,6}$, Rong Liu ${ }^{9}$, Shubin Liu ${ }^{4,7}$, Xingyan Liu ${ }^{9}$, Guangyuan Luan ${ }^{8}$, Qili $\mathrm{Mu}^{5,6}$, Binbin $\mathrm{Qi}^{4,7}$, Jie Ren ${ }^{8}$, Zhizhou Ren ${ }^{9}$, Xichao Ruan ${ }^{8}$, Zhaohui Song ${ }^{10}$, Yingpeng Song ${ }^{5,6}$, Hong Sun ${ }^{5,6}$, Kang Sun ${ }^{5,6,11}$, Xiaoyang Sun ${ }^{5,6,11}$, Zhijia Sun ${ }^{4,5,6}$, Zhixin Tan $^{5,6}$, Hongqing Tang ${ }^{8}$, Xinyi Tang ${ }^{4,7}$, Binbin Tian ${ }^{5,6}$, Lijiao Wang ${ }^{5,6,11}$, Pengcheng Wang ${ }^{5,6}$, Qi Wang ${ }^{8}$, Taofeng Wang ${ }^{13}$, Zhaohui Wang ${ }^{8}$, Jie Wen ${ }^{9}$, Zhongwei Wen ${ }^{9}$, Qingbiao $\mathrm{Wu}^{5,6}$, Xiaoguang $\mathrm{Wu}^{8}$, Xuan $\mathrm{Wu}^{5,6}$, Likun Xie ${ }^{4,12}$, Yiwei Yang ${ }^{9}$, Li $\mathrm{Yu}^{5,6}$, Tao $\mathrm{Yu}^{4,7}$, Yongji $\mathrm{Yu}^{5,6}$, Linhao Zhang ${ }^{5,6,11}$, Qiwei Zhang ${ }^{8}$, Xianpeng Zhang ${ }^{10}$, Yuliang Zhang ${ }^{5,6}$, Zhiyong Zhang ${ }^{4,7}$, Yubin Zhao ${ }^{5,6}$, Luping Zhou ${ }^{5,6,11}$, Zuying Zhou ${ }^{8}$, Danyang Zhu ${ }^{4,7}$, Kejun Zhu ${ }^{4,5}$, and Peng Zhu ${ }^{5,6}$

${ }^{1}$ State Key Laboratory of Nuclear Physics and Technology, School of Physics, Peking University, Beijing 100871, China

${ }^{2}$ Frank Laboratory of Neutron Physics, JINR, Dubna 141980, Russia

${ }^{3}$ Nuclear Research Centre, National University of Mongolia, Ulaanbaatar, Mongolia

${ }^{4}$ Sate Key Laboratory of Particle Detection and Electronics

${ }^{5}$ Institute of High Energy Physics, Chinse Academy of Sciences (CAS), Beijing 100049, China

${ }^{6}$ Spallation Neutron Science Center, Dongguan 523803, China

${ }^{7}$ Department of Modern Physics, University of Science and Technology of China, Hefei 230026, China

${ }^{8}$ Key Laboratory of Nuclear Data, China Institute of Atomic Energy, Beijing 102413, China

${ }^{9}$ Institute of Nuclear Physics and Chemistry, China Academy of Engineering Physics, Mianyang 621900, China

${ }^{10}$ Northwest Institute of Nuclear Technology, Xi' an 710024, China

${ }^{11}$ University of Chinese Academy of Sciences, Beijing 100049, China

${ }^{12}$ Department of Engineering and Applied Physics, University of Science and Technology of China, Hefei 230026, China

${ }^{13}$ Beihang University, Beijing 100083, China

\begin{abstract}
In the past two decades cooperating with Frank Laboratory of Neutron Physics (FLNP), Joint Institute for Nuclear Research (JINR) measurements of $(n, \alpha)$ reaction cross sections for ${ }^{6} \mathrm{Li},{ }^{10} \mathrm{~B},{ }^{25} \mathrm{Mg},{ }^{39} \mathrm{~K}$, ${ }^{40} \mathrm{Ca},{ }^{54,56,57} \mathrm{Fe},{ }^{58} \mathrm{Ni},{ }^{63} \mathrm{Cu},{ }^{64,67} \mathrm{Zn},{ }^{95} \mathrm{Mo},{ }^{143} \mathrm{Nd}$ and ${ }^{147,149} \mathrm{Sm}$ nuclei were performed in the $\mathrm{MeV}$ neutron energy region based on the 4.5 MV Van de Graaff accelerator at Peking University. In recent years, our measurements were extended in three aspects. Firstly, measurements were expanded from two-body reactions to three-body reactions such as ${ }^{10} \mathrm{~B}(n, t 2 \alpha)$. Secondly, the neutron energy region was extended from below $8 \mathrm{MeV}$ to $8-11$ $\mathrm{MeV}$ by using the HI-13 tandem accelerator of China Institute of Atomic Energy (CIAE), with which cross sections of ${ }^{54,56} \mathrm{Fe}(n, \alpha){ }^{53,51} \mathrm{Cr}$ reactions were measured. Thirdly, based on the newly-built China Spallation Neutron Source (CSNS) Back-n WNS (White Neutron Source), differential and angle-integrated cross sections for ${ }^{6} \mathrm{Li}(n, t)$ and ${ }^{10} \mathrm{~B}(n, \alpha)$ reactions were measured in the neutron energy region from $1 \mathrm{eV}$ to $3 \mathrm{MeV}$.
\end{abstract}

\section{Introduction}

Neutron-induced light-charged particle emission reactions $[(n, l c p)$ reactions] are important in nuclear engineering (such as the neutron standards, fusion reactors, and accelerator driven system) and basic research (such as the study of nuclear reaction mechanism, and the synthesis of isotopes in early universe). However, measurement data are scarce with large uncertainties (except for the results at thermal energies) because the measurements of these reactions are rather difficult. The main challenges for the measurement are as follows: a) the cross sections are often small, b) the intensity of the neutron sources are limited, c) highly enriched isotopic materials are needed, d) thicknesses of the samples are limited by the short ranges of the charged ejectiles, and e) the backgrounds are strong. By applying the techniques solving the problems above, we have been measuring the $(n, l c p)$ reactions for years based on different neutron sources.

*e-mail: guohuizhang@pku.edu.cn 
Table 1. The measured $(n, \alpha)$ reactions based on the $4.5 \mathrm{MV}$ Van de Graaff accelerator at Peking University.

\begin{tabular}{lll}
\hline Reaction & $E_{n}(\mathrm{MeV})$ & Reference \\
\hline${ }^{6} \mathrm{Li}(n, t)$ & $1.05,1.54,1.85,2.25,2.67$, & {$[1-4]$} \\
& $3.67,4.42$ & {$[5,6]$} \\
\hline${ }^{10} \mathrm{~B}(n, \alpha)$ & $4.0,5.0$ & {$[7,8]$} \\
\hline${ }^{10} \mathrm{~B}(n, t 2 \alpha)(n, \alpha)$ & $4.0,4.5,5.0$ & {$[9]$} \\
\hline${ }^{25} \mathrm{Mg}(n, \alpha)\left(n, \alpha_{0}\right)$ & $4.0,4.5,5.0,5.5,6.0$ & {$[10,11]$} \\
\hline${ }^{39} \mathrm{~K} /{ }^{40} \mathrm{Ca}(n, \alpha)$ & $4.5,5.5,6.5 / 5.0,6.0$ & {$[12]$} \\
\hline${ }^{40} \mathrm{Ca}\left(n, \alpha_{0}\right)\left(n, \alpha_{12}\right)$ & $4.0,4.5,5.0,5.5,6.0,6.5$ & \\
$\left(n, \alpha_{345}\right)$ & & {$[13,14]$} \\
\hline${ }^{54} \mathrm{Fe} /{ }^{56} \mathrm{Fe} /{ }^{57} \mathrm{Fe}$ & $4.0,4.5,5.5,6.5 / 5.5,6.5 /$ & \\
$(n, \alpha)$ & $5.0,5.5,6.0,6.5$ & {$[15]$} \\
\hline${ }^{54,56} \mathrm{Fe}(n, \alpha)$ & $5.5,7.7$ & {$[16]$} \\
\hline${ }^{58} \mathrm{Ni}(n, \alpha)$ & $6.0,7.0$ & {$[14]$} \\
\hline${ }^{63} \mathrm{Cu}(n, \alpha)$ & $5.0,5.5,6.0,6.5$ & {$[17,18]$} \\
\hline${ }^{64}, \mathrm{Zn}(n, \alpha)$ & $2.54,4.00,5.03,5.50,5.95$ & {$[19,20]$} \\
\hline${ }^{67} \mathrm{Zn}(n, \alpha)\left(n, \alpha_{0}\right)$ & $4.0,5.0,6.0$ & {$[21]$} \\
\hline${ }^{95} \mathrm{Mo}(n, \alpha)$ & $4.0,5.0,6.0$ & {$[22]$} \\
\hline${ }^{143} \mathrm{Nd}(n, \alpha)$ & $4.0,5.0,6.0$ & {$[22,23]$} \\
\hline${ }^{147} \mathrm{Sm}(n, \alpha)$ & $5.0,6.0$ & {$[24,25]$} \\
\hline${ }^{149} \mathrm{Sm}(n, \alpha)$ & $4.5,5.0,5.5,6.0,6.5$ & \\
\hline
\end{tabular}

\section{Two decades' measurements based on the 4.5 MV Van de Graaff accelerator at Peking University}

Cooperating with Frank Laboratory of Neutron Physics (FLNP), Joint Institute for Nuclear Research (JINR), and based on the 4.5 MV Van de Graaff accelerator at Peking University, we have measured a series of ( $n$, $\alpha$ ) reactions, including light, medium-mass and heavy nuclei. Twin gridded ionization chambers (GICs) were used as detectors for charged particles with a large solid angle (nearly $4 \pi$ ) and high efficiency ( $100 \%)$. NIM block electronics and home-made data acquisition system were employed. The neutron energy range was from 1.0 to 7.7 $\mathrm{MeV}$.

Combining measurements with theoretical analysis, systematic results including angular differential cross sections and/or cross sections were obtained. Details of the reaction channels, the energy points and related publications are listed in Table 1.

\section{Recent developments}

\subsection{Measurement of the three-body reaction}

Based on the 4.5 MV Van de Graaff accelerator at Peking University, measurement of cross sections of the three-body reaction ${ }^{10} \mathrm{~B}(n, t 2 \alpha)$ was performed [7, 8]. A thin-backing ${ }^{10} \mathrm{~B}$ sample was prepared, and the ${ }^{10} \mathrm{~B}$ atom number in the sample was measured relative to the ${ }^{6} \mathrm{Li}$ atom number in the standard ${ }^{6} \mathrm{LiF}$ sample using thermalneutron-induced reactions of ${ }^{10} \mathrm{~B}$ and ${ }^{6} \mathrm{Li}$. Digital data acquisition system based on wave form digitizers and LABVIEW software were developed. Forward as well as backward signals of grids and anodes of the twin GIC were recorded. Methods of anode-grid and forward-backward coincidences were used to select the valid events. Cross sections of the three-body reaction ${ }^{10} \mathrm{~B}(n, t 2 \alpha)$, as well as the two-body reaction ${ }^{10} \mathrm{~B}(n, \alpha){ }^{7} \mathrm{Li}$ were measured simultaneously at 4.0, 4.5 and $5.0 \mathrm{MeV}[7,8]$.

\subsection{Measurements based on the $\mathrm{HI}-13$ tandem accelerator at CIAE}

In addition to the measurements of the ${ }^{56,54} \mathrm{Fe}(n$, $\alpha)^{53,51} \mathrm{Cr}$ cross sections at the Van de Graaff accelerator at Peking University, neutron energy range was extended to 8 - $11 \mathrm{MeV}$ based on the HI-13 tandem accelerator of China Institute of Atomic Energy (CIAE). Using deuterium gas target and d-d reaction as the neutron source, cross sections of the ${ }^{56,54} \mathrm{Fe}(n, \alpha){ }^{53,51} \mathrm{Cr}$ reactions were measured at $8.5,9.5$ and $10.5 \mathrm{MeV}$ [15] in which region the measurement results are scarce with big discrepancies among different experiments and evaluations. Since the neutron source was not mono-energetic in this region, the neutron energy spectra were measured using an EJ309 detector by unfolding the measured pulse height spectra [26]. The interference of the low energy neutrons was corrected. A shoulder structure was observed near $10 \mathrm{MeV}$ in both excitation functions of the ${ }^{56,54} \mathrm{Fe}(n, \alpha){ }^{53,51} \mathrm{Cr}$ reactions [15] and was analyzed.

\subsection{Measurements based on CSNS Back-n WNS}

China Spallation Neutron Source (CSNS) is the first pulsed spallation neutron source in China and it was put to service in March, 2018. An associated white neutron source (WNS) exploiting the back-streaming neutrons (Back-n) was built for nuclear data measurement [27]. ${ }^{6} \mathrm{Li}(n, t){ }^{4} \mathrm{He}$ and ${ }^{10} \mathrm{~B}(n, \alpha){ }^{7} \mathrm{Li}$ reactions were selected as the first and the second ( $n, l c p)$ reactions to be measured. Measurements were performed at the Endstation 1 (ES\#1) along the Back- $n$ beam line. The proton beam power during measurements was $\sim 20 \mathrm{~kW}$. The repetition frequency of the proton beam was $25 \mathrm{~Hz}$ (double-bunch mode).

Enriched double ${ }^{6} \mathrm{LiF}$ and double ${ }^{10} \mathrm{~B}$ samples $50 \mathrm{~mm}$ in diameter were prepared. The detector system used for the measurement was the Light charged Particle Detector Array (LPDA), which mainly consisted of 15 silicon detectors $\left(2.0 \times 2.5 \mathrm{~cm}^{2}\right.$, with detection angles ranging from $19.2^{\circ}$ to $160.8^{\circ}$ ) in a vacuum chamber. Based on the CSNS 
Back-n WNS , and the LPDA system, the ${ }^{6} \mathrm{Li}(n, t)^{4} \mathrm{He}$ and ${ }^{10} \mathrm{~B}(n, \alpha){ }^{7} \mathrm{Li}$ reactions were measured. The beam durations for measurement of the ${ }^{6} \mathrm{Li}(n, t){ }^{4} \mathrm{He}$ and ${ }^{10} \mathrm{~B}(n, \alpha){ }^{7} \mathrm{Li}$ reactions were $196 h$ and $357 h$, respectively.

Measurement data are under analysis and preliminary results are obtained. For the ${ }^{6} \mathrm{Li}(n, t)^{4} \mathrm{He}$ reaction, differential cross sections at 80 neutron energies were measured from $1 \mathrm{eV}$ to $3 \mathrm{MeV}$ (selected preliminary results are presented in Fig.1). For the ${ }^{10} \mathrm{~B}(n, \alpha)^{7} \mathrm{Li}$ reaction, differential cross sections at 67 neutron energies were measured between $1 \mathrm{eV}$ and $2.0 \mathrm{MeV}$ (selected preliminary results are presented in Fig.3). For the ${ }^{10} \mathrm{~B}\left(n, \alpha_{0}\right){ }^{7} \mathrm{Li}$ and ${ }^{10} \mathrm{~B}(n$, $\left.\alpha_{1}\right)^{7} \mathrm{Li}$ reactions, differential cross sections were measured at 59 neutron energies between $1 \mathrm{eV}$ and $1 \mathrm{MeV}$ (selected preliminary results are presented in Figs. 5 and 7, respectively). Moreover, angle-integrated cross sections for the reactions above are also obtained through integration. Preliminary results of the present cross sections for the ${ }^{6} \mathrm{Li}(n$, $t{ }^{4} \mathrm{He},{ }^{10} \mathrm{~B}(n, \alpha){ }^{7} \mathrm{Li},{ }^{10} \mathrm{~B}\left(n, \alpha_{0}\right)^{7} \mathrm{Li}$ and ${ }^{10} \mathrm{~B}\left(n, \alpha_{1}\right){ }^{7} \mathrm{Li}$ reactions compared with those of other measurements and evaluations are shown in Figs.2, 4, 6 and 8, respectively. Present data are subject to further corrections.

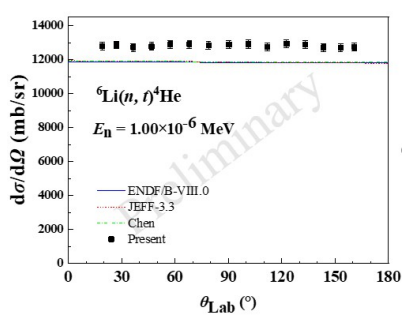

(a) $E_{n}=1.00 \times 10^{-6} \mathrm{MeV}$

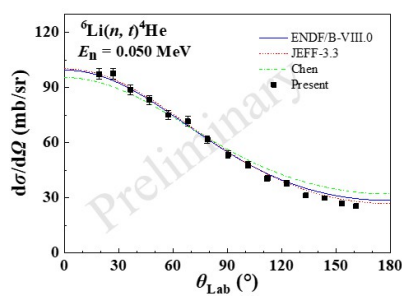

(c) $E_{n}=0.050 \mathrm{MeV}$

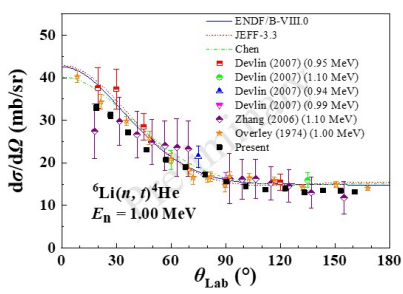

(e) $E_{n}=1.00 \mathrm{MeV}$

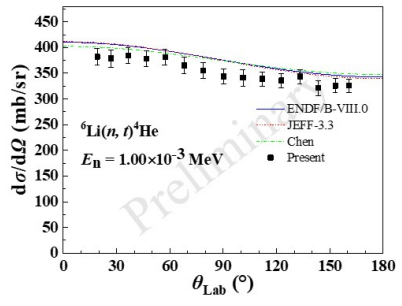

(b) $E_{n}=1.00 \times 10^{-3} \mathrm{MeV}$

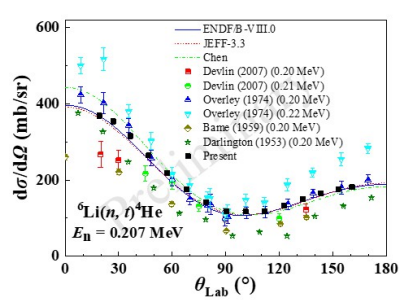

(d) $E_{n}=0.207 \mathrm{MeV}$

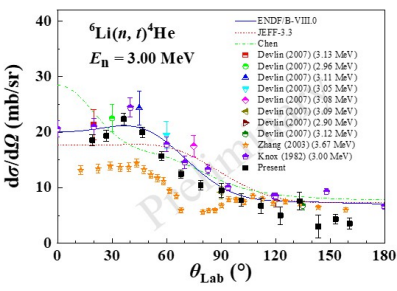

(f) $E_{n}=3.00 \mathrm{MeV}$
Figure 1. Selected preliminary results of differential cross sections for the ${ }^{6} \mathrm{Li}(n, t)^{4} \mathrm{He}$ reaction.

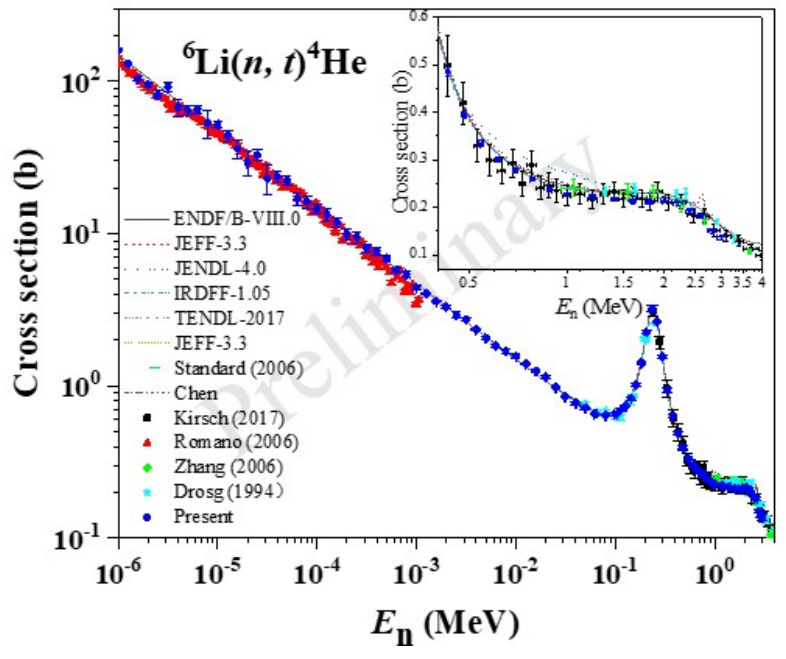

Figure 2. Preliminary results of present cross sections for the ${ }^{6} \mathrm{Li}(n, t)^{4} \mathrm{He}$ reaction compared with those of other measurements and evaluations.

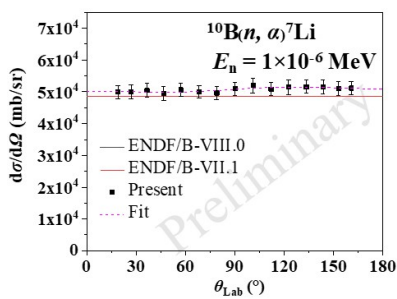

(a) $E_{n}=1.00 \times 10^{-6} \mathrm{MeV}$

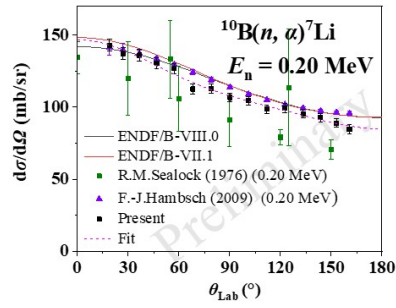

(c) $E_{n}=0.20 \mathrm{MeV}$

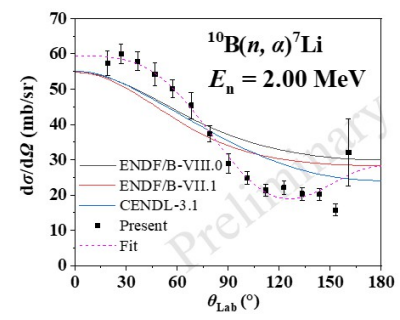

(e) $E_{n}=2.00 \mathrm{MeV}$

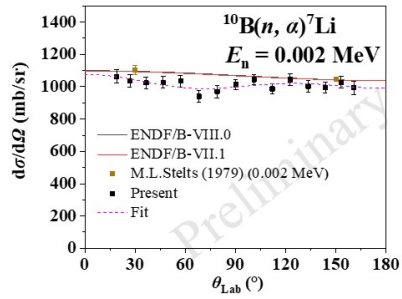

(b) $E_{n}=0.002 \mathrm{MeV}$

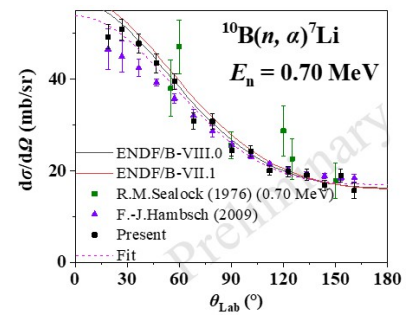

(d) $E_{n}=0.70 \mathrm{MeV}$
Figure 3. Selected preliminary results of differential cross sections for the $\left.{ }^{10} \mathrm{~B}(n, \alpha)\right)^{7} \mathrm{Li}$ reaction. 


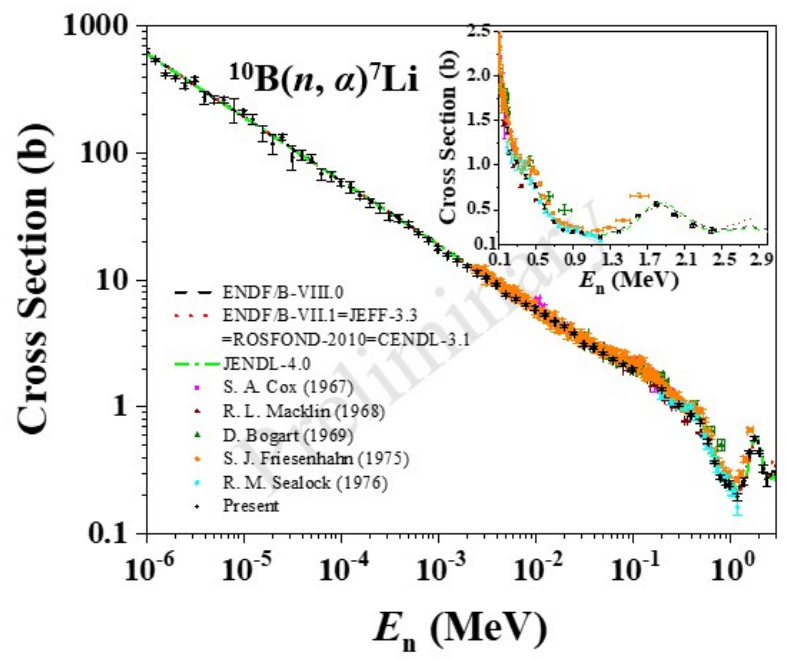

Figure 4. Preliminary results of present cross sections for the ${ }^{10} \mathrm{~B}(n, \alpha){ }^{7} \mathrm{Li}$ reaction compared with those of other measurements and evaluations.

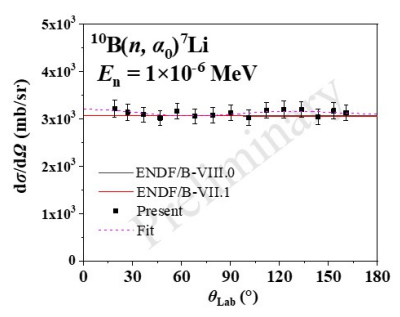

(a) $E_{n}=1.00 \times 10^{-6} \mathrm{MeV}$

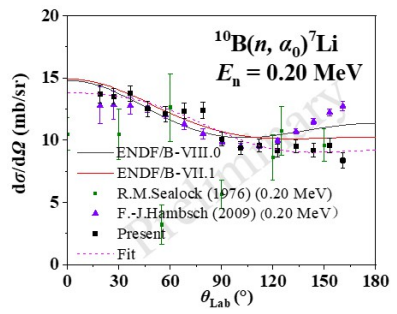

(c) $E_{n}=0.20 \mathrm{MeV}$

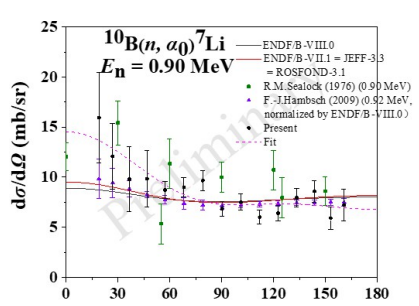

(e) $E_{n}=0.90 \mathrm{MeV}$

Figure 5. Selected preliminary results of differential cross sections for the ${ }^{10} \mathrm{~B}\left(n, \alpha_{0}\right)^{7} \mathrm{Li}$ reaction.

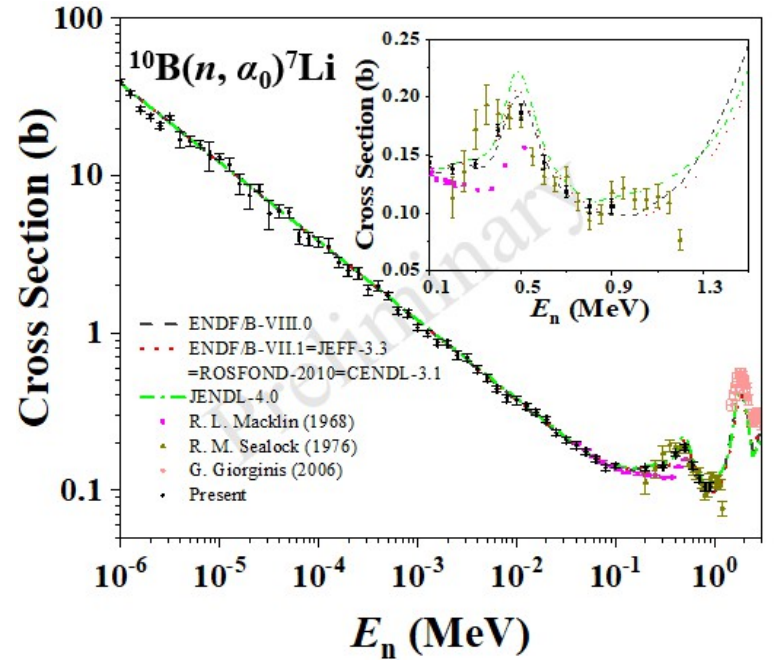

Figure 6. Preliminary results of present cross sections for the ${ }^{10} \mathrm{~B}\left(n, \alpha_{0}\right){ }^{7} \mathrm{Li}$ reaction compared with those of other measurements and evaluations.

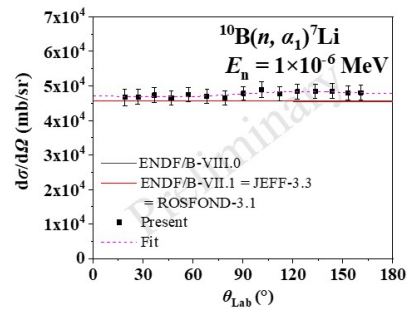

(a) $E_{n}=1.00 \times 10^{-6} \mathrm{MeV}$

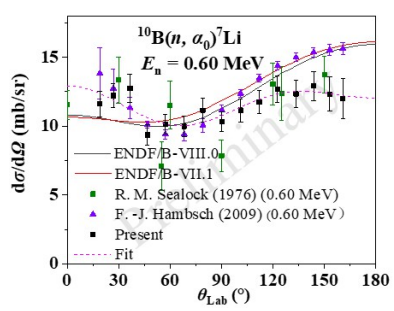

(d) $E_{n}=0.60 \mathrm{MeV}$

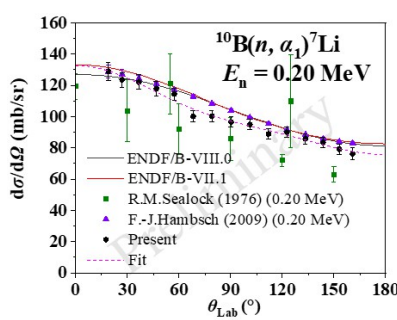

(c) $E_{n}=0.20 \mathrm{MeV}$

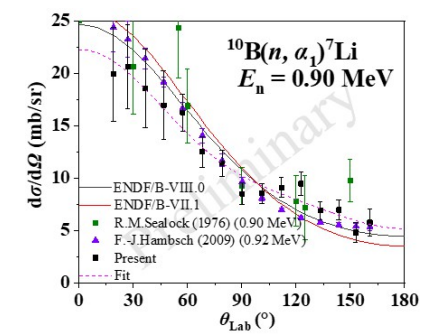

Figure 7. Selected preliminary results of differential cross sections for the ${ }^{10} \mathrm{~B}\left(n, \alpha_{1}\right)^{7} \mathrm{Li}$ reaction. (e) $E_{n}=0.90 \mathrm{MeV}$

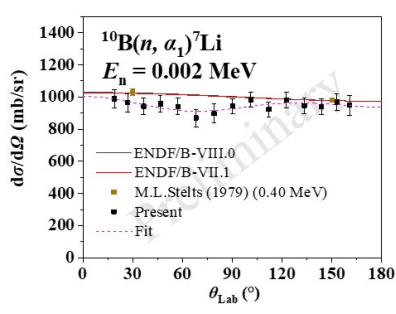

(b) $E_{n}=0.002 \mathrm{MeV}$

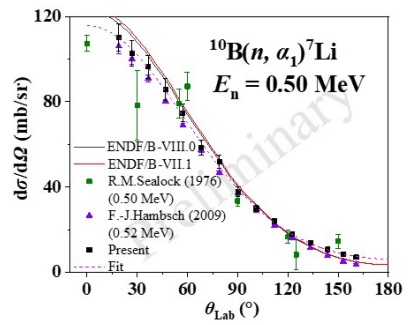

(d) $E_{n}=0.50 \mathrm{MeV}$ 


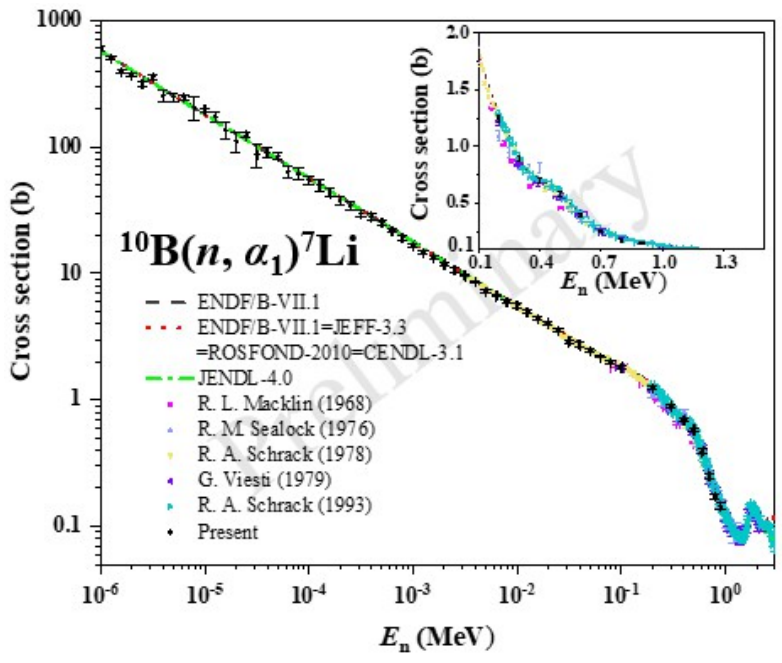

Figure 8. Preliminary results of present cross sections for the ${ }^{10} \mathrm{~B}\left(n, \alpha_{1}\right){ }^{7} \mathrm{Li}$ reaction compared with those of other measurements and evaluations.

\section{Conclusion and outlook}

For more than 20 years, we have been engaged in the measurements of the $(n, l c p)$ reactions based on the 4.5 MV Van de Graaff accelerator at Peking University. In recent years, we extended our measurements based on the HI-13 tandem accelerator of CIAE and on CSNS. A series of measurements have been performed and systematical results obtained. Further measurements are planned, such as the ${ }^{58,60,61} \mathrm{Ni}(n, \alpha)$ reactions and the $n-p$ and $n$ - $d$ scattering reactions.

\section{References}

[1] G. Zhang et al., Nucl Sci Eng 134, 312-316(2000)

[2] G. Zhang et al., Nucl Sci Eng 143, 86-89(2003)

[3] G. Zhang et al., Nucl Sci Eng 153, 41-45(2006)

[4] G. Zhang et al., Nucl Inst Meth A 566, 615-621(2006)

[5] G. Zhang et al., Appl Rad Isot 66, 1427-1430(2008)

[6] G. Zhang et al., Chin Phys Lett 28, 082801(2011)

[7] Z. Wang et al., Phys Rec C 96, 044621(2017)

[8] Z. Wang et al., Phys Rec C 96, 044620(2017)

[9] Yu. M. Gledenov et al., Phys Rec C 98, 034605(2018)

[10] X. Zhang et al., Phys Rec C 61, 054607(2000)

[11] X. Zhang et al., Nucl Sci Eng 134, 89-96(2000)

[12] J. Han et al., Eur Phys J A 51, 12(2015)

[13] Z. Wang et al., Phys Rev C 92, 044601(2015)

[14] Yu. M. Gledenov et al., Phys Rev C 89, 064607(2014)

[15] H. Bai et al., Phys Rev C 99, 024619(2019)

[16] J. Fan et al., INDC(CPR)-034/L 13, 1-9(1995)

[17] G. Zhang et al., Nucl Sci Eng 156, 115-119(2007)

[18] G. Zhang et al., Nucl Sci Eng 160, 123-128(2008)

[19] G. Zhang et al., Phys Rev C 82, 054619(2010)

[20] G. Zhang et al., Eur Phys J A 43, 1-4(2010)

[21] G. Zhang et al., Appl Rad Isot 68, 180-183(2010)

[22] Yu. M. Gledenov et al., Phys Rev C 80, 044602(2009)

[23] G. Zhang et al., Appl Rad Isot 67, 46-49(2009)

[24] Yu. M. Gledenov et al., Phys Rev C 82, 014601(2010)

[25] G. Zhang et al., Phys Rev Lett 107, 252502(2011)

[26] H. Bai et al., Nucl Inst Meth A 886, 109-118(2018)

[27] Y. Chen et. al., Eur Phys J A 55, 115(2019) 